\title{
Fatigue Crack Shielding and Deflection in Plain Bearings under Large Scale Yielding
}

\author{
A. Burke-Veliz ${ }^{\dagger}$, S. Syngellakis, P.A.S. Reed.
}

School of Engineering Sciences, University of Southampton, Southampton SO17 1BJ, United Kingdom

\begin{abstract}
Multi-layered bearing systems used in the automotive industry show shielding and antishielding effects that reduce or amplify the crack driving force under large scale yielding conditions. Using finite element analysis, it is shown that shielding in such systems results in path deflection and bifurcation despite the absence of mixed-mode loading. As the crack approaches a stiff layer, the tangential strains measured around a blunted crack tip model show a maximum corresponding to the direction of crack propagation. The distribution of such strains indicates the effect of shielding and the likelihood of the tip to deflect or bifurcate. The suitability of bi-layer and tri-layer bearing architectures is assessed through crack path and respective crack driving force predictions.
\end{abstract}

Keywords: Crack growth, Surface layers, Bearing failure, Automotive design.

\section{Introduction}

Fracture and fatigue failure has been extensively studied within both academic and industrial environments due the cost involved in rectifying service failures, production of spare parts,

\footnotetext{
${ }^{\dagger}$ Corresponding author: Tel: (44) 238059 3392; fax: (44) 2380594813

Email: allan.burke@itesm.mx
} 
service intervals and lead times. New materials and design processes have supported the introduction of more demanding service conditions. This trend is evident in the automotive industry where power efficiency and component packaging drive the requirement for smaller, more lightweight and durable components. The design of plain bearings is one case in point, due to their importance in the powertrain system and their strategic position within the engine. In machine design, more emphasis is usually given to heavier components such as connecting rods, pistons or shafts [1]. However, the bearing design process is complex and involves assessment of manufacture, assembly and service conditions. The analysis of bearings under such conditions is especially interesting and challenging since it relies on predictions of the hydrodynamic oil film pressure and the associated housing deformation. As shown in Figure 1, the film pressure has steep gradients causing mixed-mode loading and, consequently, may lead to complex crack growth patterns in the lining.

Bearing performance has been enhanced through the use of multi-layered architectures and the development of lining materials with favorable mechanical properties. These multilayered systems provide the required compromise between stiffness and tribological performance through a stiff backing layer and a conformable and low-friction lining. An additional attribute of multi-layered structures is the shielding effect that reduces the growth rate as cracks approach stiffer layers [2-5], thus increasing service life. The opposite effect is observed as a crack approaches a more compliant layer. Bifurcation events have also been observed in bi-layered architectures in compact specimens of plastically-mismatched materials [4], especially a high levels of loading.

Most of previous work concerns small scale yielding conditions (SSY) in elastically or plastically mismatched materials, but not both. Crack growth in multi-layered architectures subjected to cyclic loading has been studied through an analytical model [3], which identified 
a further shielding effect arising from CTOD dropping as a result of crack closure since the reversed plasticity should not be affected by the layered architecture. Related numerical work based on predefined paths has been carried out using various techniques, such as cohesive elements [6], remeshing [7] and configurational forces [8], to account for crack extension. Such techniques have been widely applied to cracked specimens of homogeneous and inhomogeneous materials subjected to monotonic loading. The study of crack growth under cyclic loading is often restricted to few or representative load cycles and involves greater theoretical challenges for inhomogeneous materials as explained by Groh et al [9].

Flat strip specimens with a multi-layered composition identical to that of formed bearings have been tested under cyclic three-point bending conditions. This architecture consists of elastically and plastically mismatched materials. Crack shielding has been shown to cause deflection and bifurcation in such specimens despite the absence of far-field mixed-mode loading [10, 11], as shown in Figure 2(a). J integral estimations based on finite element modeling of the three-point bending tests [10] reported a strong tendency for highly deflected bifurcated crack paths, corresponding to a sharply increasing $\mathrm{J}$ integral, representing the crack driving force as the crack tip approaches a stiffer layer. This was in accordance with experimental results and explained in terms of the crack following the path that offers the least resistance or maximizes the CDF.

Crack path deflection has been extensively studied in brittle and ductile materials [12-14]; the prediction of crack deflection and bifurcation has been pursued due to the importance of such events in shaping the crack path and affecting the value of CDF. Detailed observation of tested components has identified coalesced cracks, as schematically shown in Figure 1, that release lining fragments under bearing service conditions; similar behavior has been observed in flat strip specimens of the same architecture under simpler three-point bending tests [15]. It 
is assumed here that deflected or bifurcated crack paths may be responsible for such failure mechanisms $[16,17]$.

Deflected crack growth under mode I-dominant, monotonic loading can be consistently simulated using various deflection criteria. Such applications to engineering problems have often used the criterion based on closed-form solutions for the maximum tangential stress around the crack tip [18]; this is obtained in the context of LEFM. However, no single approach to crack deflection prediction has been tested for optimum performance over a full range of mixed-mode loading.

The objective of this work is to explain further the shielding and bifurcation phenomena leading to fatigue failure in tri-layer material systems. The methodology is based on the evaluation of the crack tip opening displacement (CTOD), an alternative CDF parameter suitable to large-scale yielding (LSY) conditions. The crack path and respective CDF evolution is investigated for growing cracks in multi-layer flat strips with mismatched mechanical properties that are subjected to three-point bending causing extensive plasticity. The state of stress around the crack tip is studied to assess the conditions that promote crack deflection and bifurcation despite the absence of far-field mixed-mode loading. Thus, it becomes possible to assess the impact of multi-layered architectures on crack growth under LSY, which also occurs in plain bearings under service conditions. The focus of the numerical investigation is tri-layer architectures in order to assess the influence of a compliant interlayer, which is found in some plain bearing designs as it provides a protective deposition layer for the steel and improves the bonding between layers. An equivalent bilayer system is also analyzed for comparison purposes. 


\section{Methodology}

The first part of this numerical investigation is concerned with straight cracks of variable length in a tri-layer system in order to assess the combined effect of anti-shielding and shielding. A bi-layer architecture is also analyzed for comparison with a pure shielding case at this stage. For each assumed crack length, crack tip strains and CTOD are recorded for further assessment of possible deflection and shielding.

In the second part, crack growth along deflected and bifurcated paths is simulated to investigate the experimentally observed tendency of the cracks to grow parallel to the layers' orientation and the effect of crack deflection on CDF estimates. These simulations are based on an automatic crack extension routine based on the estimates of crack tip tangential strains (CTTS) so that the maximum tangential strain (MTSN) deflection criterion could be implemented using ANSYS Parametric Design Language (APDL) [19]. This routine extended the crack by small straight segments with gradually changing orientation specified by angle $\beta$, as indicated in Figure 3(b), determined according to the numerical maximum along the estimated CTTS curves. The magnitude and distribution of the latter depend on the extent of plasticity and the layered architecture.

Using the results from the analyses on straight cracks, conditions for initial deflection were established. With regard to bifurcation, in particular, a parametric study was performed to assess the influence of the crack length $a$ before bifurcation on the CDF at the tip of small crack kinks at angles $\theta$ to the original straight path, as shown in Fig. 3(c). The extension step in the crack growth analysis was gradually reduced until the forecasted path converged to a stable line. 
The models used at both stages are shown in Figure 3. The thicknesses of the lining, interand backing layers are $t_{\mathrm{L} 1}=0.38 \mathrm{~mm}, t_{\mathrm{i}}=0.04 \mathrm{~mm}$ and $t_{\mathrm{b}}=1.8 \mathrm{~mm}$, respectively. For the bilayer, the lining thickness was taken equal to $t_{\mathrm{L} 2}=t_{\mathrm{L} 1}+t_{\mathrm{i}}=0.42 \mathrm{~mm}$. The analyses are based on two-dimensional finite element (FE) models of a multi-layered flat strip and appropriate fracture mechanics concepts applicable to monotonic and fatigue loading. The three-point bending loading is applied to replicate the experimental conditions in previous fatigue tests $[11,15,20]$; this generates pure opening mode at the central section of the strip just opposite to the applied load $\mathrm{P}$ as shown in Figure 3. Bifurcated and deflected paths have been experimentally observed to evolve from originally straight cracks at this location.

The performed FE analyses were based on the general-purpose software ANSYS 11.0 [19] using re-meshing schemes around straight or deflected propagating cracks. Elasto-plastic material models were used consistent with the values for the Young's modulus $E$ and the yield stress $\sigma_{Y}$ presented in Figure 2(b) [21]. The respective true stress-true strain curves are shown in Figure 4. The ANSYS analysis procedure is set up to re-calculate the compliance matrix after each converged iteration in the solution process incorporating the effect of large deformations. In preliminary simulations of three-point bending tests, the region around the crack tip was meshed using parametric quarter-point elements [22] in spider-web configurations. Such elements however showed excessive distortions under high loads causing large plastic strains, especially in the compliant interlayer, which requires an even more refined mesh due to its small thickness. A blunted tip [23], modeled as a semi-circle of very small radius, was adopted to reliably predict large crack tip deformations. Refined FE meshes provided detailed information on the crack tip stresses, strains and displacements, which were read relative to a local polar frame of reference with origin at the centre of the semi-circle. 
The crack tip opening displacement (CTOD) is adopted in this work as CDF parameter over the $\mathbf{J}$ integral as it represents more accurately the local crack tip conditions. Although the $\mathbf{J}$ integral can be estimated so that it also represents local conditions $[5,7,8,10]$, it is generally adopted as a global measure and as such, it requires a more intricate implementation in the case of bi-materials under large scale yielding. The CTOD is also theoretically better suited for characterizing cyclic loading in the context of a fatigue crack propagation law and was here obtained as the relative displacement of two nodes placed at the two ends of the blunted tip semi-circle.

Previous work on deflected crack paths [24] concluded that a strain based approach represents more closely the fatigue process. This justified the choice of MTSN [12] as the criterion for assessing the direction of crack deflection; its validity was further assessed by applying it together with other criteria to the mixed-mode crack growth experiments by Mageed and Pandey [25]. MTSN provided the best correlation with the experimental results in comparison with the maximum tangential stress and crack tip deflection vector criteria, which are also relevant to LSY. Its implementation was carried out using the FE estimates of tangential strain at the nodes placed at $5^{\circ}$ intervals around the blunted tip. To obtain an estimate at any point along the blunted tip, a second order interpolation was adopted using the crack tip tangential strains (CTTS) at three adjacent nodes.

\section{Results and discussion}

\subsection{Straight cracks}

The numerical study of straight stationary cracks provides an insight into the shielding and anti-shielding mechanisms in multi-layered architectures. The introduction of the interlayer into the bearing design improves the bonding between lining and backing but does not play a 
significant role in the overall stiffness of the component under bending since its thickness is just $1.8 \%$ of the total bearing thickness, while the lining represents $9.5 \%$.

Figure 5 shows the CDF evolution as cracks approach dissimilar layers in the tri-layer system. In this as well as all subsequent graphs, the CTOD results are plotted against the ratio of the crack length a to the lining thickness $t_{\mathrm{L} 2}$ of the bi-layer system as shown in Figure 3(b). The shielding and amplification trends observed are consistent with those reported in similar previous investigations based on J-integral estimates of systems with principally plastic [7] but also elastic [10] mismatch. The amplification effect is noted when the crack approaches the more compliant interlayer, and shielding when the crack approaches the stiffer backing within the interlayer. The increase of the applied load $\mathrm{P}$ and the subsequent spread of plasticity led to greater mismatches between layers, which caused increased CTOD gradients and therefore more pronounced shielding and amplification at lower crack lengths.

For the bi-layer architecture, CTOD is predicted to decrease as the crack tip approaches the stiffer steel layer, as shown in Figure 6. The shielding effect is also influenced in this case by the magnitude of the applied load since, as the load increases, the spread of plasticity into the backing layer reduces the property mismatch between the layers and, as a consequence, shifts the CTOD maximum closer to the lining/backing interface.

The crack tip tangential strains (CTTS) play an important role in this study as indicators of possible crack deflection according to the MTSN criterion. Their predictions showed variable patterns depending on the applied load, crack length, layer in which the crack tip is located and architecture analyzed. The CTTS contours around the blunted tip within the lining of the tri-layer architecture are shown in Figure 7; it is noted that the maximum CTTS value remains at the zero-degree position corresponding to the direction normal to the layers. This 
direction should therefore be the preferred instantaneous direction of propagation in accordance with respective CTOD profiles and experimental observations. In contrast, the CTTS contours in the bi-layer system have different trends for crack lengths above $0.45 t_{\mathrm{L} 2}$ as shown in Figure 8. There appears to be a spread of CTTS values quite close to the maximum over a range of angles indicating a tendency for possible deflection along a direction within that range. As the crack length increases further, this zone of possible deflection is divided into two symmetrically located branches of maximum CTTS values, confirming that deflection should have occurred.

Although the CTTS maximum is clearly distinguishable for straight cracks within the lining of the tri-layer bearing, especially at higher loads, it actually becomes less sharp as the crack tip approaches the lining-interlayer boundary. This is against expectations as it contradicts the respective CTOD behavior described earlier (see Figure 5) indicating crack growth amplification and lower tendency for deflection. High stress concentrations and crack tip distortion is expected to occur near a bi-material interface. The reliability of CTTS-based predictions of possible crack deflection under such conditions should be carefully examined.

The CTTS variation provides a qualitative indication of the natural likelihood of either architecture to contain bifurcated or deflected cracks. The results of this analysis are consistent with experimental observations in bearings with deflected paths having been reported in the lining of bi-layer architectures $[16,17]$, while tri-layer architectures usually display deflected or bifurcated paths within the interlayer. Crack deflection has also been observed to a lesser extent in the lining of tri-layer bearings but this can be attributed to mixed mode conditions arising from material heterogeneity. 


\subsection{Deflected paths}

The study of CTOD and CTTS for straight cracks normal to the layers revealed the shielding and anti-shielding effects and the likelihood of the crack to deflect in both architectures. The CTTS variation along the blunted tip indicated that, as the crack approaches a stiffer layer, there is a spread of CTTS values near the maximum over a range of angles so that any asymmetric feature, such as material heterogeneity or load eccentricity, can trigger a deflection from the straight path.

The crack growth simulation in the bi-layer lining confirmed the tendency for the crack to deflect, as predicted by the analysis of the flat strip with straight cracks. The predicted deflections were dependent on the extent of plasticity caused by the applied load. Under low loads, insignificant deflections were predicted by the MTSN criterion; deflections below $20^{\circ}$ were only observed under the load $\mathrm{P}=800 \mathrm{~N}$, very close to the layers interface.

The prediction of small deflections led to the consideration of deflections possibly arising from the irregular, multi-phase micro-structure of the lining material observed in tested bearings $[11,20]$. This kind of heterogeneity would modify locally the solution based on problem symmetry into a mixed-mode stress field triggering deflection. This scenario was tested in both architectures at $\mathrm{P}=200 \mathrm{~N}$ and $\mathrm{P}=800 \mathrm{~N}$ by introducing a large artificial deflection at some crack length. The corresponding results for the subsequent crack growth showed that the crack follows a preferred path, which is the one obtained according to the implemented deflection criterion and that any induced deflections only alter locally the shape of path and the respective CDF estimates; a quick return to the preferred path subsequently occurs. 
The analysis of crack growth in the tri-layer bearing lining resulted in straight paths and CDF estimates identical to the ones obtained for straight stationary cracks; small deviations noted during crack extension can be attributed to numerical errors caused by the asymmetry of the generated FE mesh. The crack growth simulation in the interlayer showed a deflected path that approached in an asymptotic manner the interlayer-backing interface, as shown in Figure 9. Figure 10 allows a comparison between CTTS patterns for straight and deflected paths. Figure 10(a) shows the tendency of the straight crack to deflect while Figure 10(b) shows the maximum CTTS becoming more pronounced along the deflected crack. This behavior was predicted under any applied load and is consistent with experimental observations. The introduction of an arbitrary induced deflection to the straight crack path in this architecture had the same effect as that described for the bi-layer system.

The tri-layer crack growth scenario displayed a tendency for the crack to propagate in the direction parallel to the interlayer rather than penetrate into the stiff backing layer. The computed CTOD-CDF along the deflected crack was found to be greater than along a straight crack normal to the interfaces. This confirmed the natural preference for a deflected crack path within the softer interlayer. As observed experimentally [17], the deflected path will grow until it coalesces with another crack and, subsequently, causes a fragment of the lining to detach. Within the interlayer, the crack tip was completely embedded within the plastic zone that extended over a significant portion of that layer enhancing the mismatch between it and the other two layers. This increased the constraint on the crack tip to move within the interlayer leading to conditions favoring deviation from problem symmetry. 


\subsection{Bifurcated paths}

The occurrence of bifurcation or branching in ductile materials has been previously related to sudden increases of the crack driving force or reductions the crack growth resistance [26]. Therefore, the extension of the analysis to the prediction of bifurcated crack growth in bearing architectures requires careful examination of the material and loading conditions encouraging such an event. The bifurcation within the interlayer of a tri-layer system shown in Figure 2(a), observed during a previous experimental investigation on the fatigue performance of bearings [11], can be attributed to a sudden drop in material resistance as soon as the crack crosses the lining-interlayer boundary. This is confirmed by the large CDF gradients noted in Figure 5 on either side of that boundary, especially at higher loads. The present numerical model of bifurcated crack growth does not account explicitly for such material changes but assumes symmetric bifurcated crack growth almost as soon as the tip enters the interlayer.

The developed parametric study in bifurcated cracks considered small crack kink lengths $d_{\mathrm{k}}=$ $0.005 t_{\mathrm{L} 2}$ within the interlayer, at angle $\theta$ to the straight path, as shown in Figure 3(c). The dependance of CDF estimates on $\theta$ was numerically obtained and examined for various values of the crack length $a$ before bifurcation. The resulting CDF variation, shown in Figure 11, confirms the importance of the proximity to a stiff layer and provides a bifurcation angle $\theta$ corresponding to a maximum $\mathrm{CDF}$ for any given crack length a. As the crack length $a$ is extended, this angle increases up to a value of $55^{\circ}$. According to Joyce et al [10], the kink angle corresponding to the maximum $\mathrm{CDF}$, represented by the J-integral, reached the value of $90^{\circ}$. Using CTOD as an initial deflection indicator did not lead to path deflections of such a magnitude. This may be due to an inherent limitation of CTOD in responding to mixed mode conditions. It is also worth mentioning that the steel backing was assumed elastic by Joyce et 
al, while here plastic deformation was allowed to develop when the load reached a certain value.

As already pointed out, bifurcation was assumed to occur as soon as the crack penetrated into the interlayer and the analysis for the bifurcated path was initiated with kink angle of $37.15^{\circ}$. Subsequent crack growth was predicted by the same process as that applied to single tip deflection analysis. The maximum tangential strain (MTSN) around the blunted tip was again used as the deflection criterion. The result of this analysis when the crack tip is very close to the backing layer is shown in Figure 12. A comparison with the corresponding result for single tip deflected crack, shown in Figure 9, indicates that the predicted crack path deflections were greater for bifurcated than for single tip propagation.

Studies on crack bifurcation have shown its impact on the values of CDF and, as a consequence, on crack growth rate, leading to longer service life. Evidently, bifurcation at early crack growth stages will extend service life more significantly. The estimated CDF values after bifurcation were reduced by between $50 \%$ and $70 \%$ for the range of loadings applied. The effect of plasticity on CDF reduction can be assessed by comparing these reductions to those predicted by Kitagawa et al [27] in elastic cracked plates under tension. The application of a methodology within the scope of EPFM is necessary when LSY conditions are present since the estimation of the CDF parameter from $\mathrm{FE}$ analyses shows a significant variation with the extent of plasticity.

\section{Concluding remarks}

The results for CTOD evolution with crack length indicate that CTOD is a well suited CDF parameter for investigating crack shielding and amplification in multi-layered systems under LSY arising from three-point-bending. These results are consistent with those previously 
published $[2,6,7,28,29]$, which mostly used the $\mathrm{J}$ integral as CDF parameter. Elasto-plastic stress analyses of bearings in operation have predicted LSY [30, 31]; CTOD could thus prove to be a reliable indicator of crack growth under such conditions.

The study of crack propagation in multi-layered systems under three-point bending helps the identification of circumstances leading to path deflection and bifurcation. In a scenario where crack bifurcation and deflection is not present, it is clear that bi-layer architectures and the associated shielding effect would retard crack growth to a great extent. However, it has been shown experimentally and numerically $[10,21]$ that as the crack approaches a stiffer interface, the tendency to deflect or bifurcate grows despite the absence of mixed-mode loading. In the context of bearing design, it should be noted that mixed-mode and variable amplitude loading is present in such components promoting crack deflection and bifurcation. Single-crack deflection in bearings is produced by the mixed-mode loading conditions; such deflections have been frequently observed in bi-layer bearing systems in the lining [31]. On the other hand, straight crack growth in the tri-layer lining is promoted as the crack tip approaches the interlayer, allowing the crack to propagate deeper into the lining and interlayer, where excessive path deflection would finally lead to the detachment of the lining after a longer service life.

Bifurcated crack patterns have been shown to be beneficial in terms of the calculated CDF and the tendency for deflection as the crack tip approaches the lining-backing interface. Nevertheless, these attributes mainly occur in the context of a single flaw. An initially straight crack in a bi-layer lining could deflect at a crack length as short as $0.5 t_{\mathrm{L} 2}$, while the straight crack in the tri-layer lining is attracted to the interlayer. The coalescence of cracks and lining particle release are clearly dependent on the random distribution of flaws around the material; nevertheless, the introduction of a soft interlayer promotes the crack growth into 
that layer across the entire lining thickness thus reducing the probability of an early lining release. Field tests in both architectures have also indicated an extended life for this tri-layer architecture along with a less significant variability in its performance.

\section{Acknowledgements}

The financial support of the Mexican Council of Science and Technology and School of Engineering Sciences is gratefully acknowledged. The training provided by and discussions with MAHLE Engine Systems was also invaluable in the development of this project.

\section{References}

[1] Grubisic V, Vulic N, Sonnichsen S. Structural durability validation of bearing girders in marine Diesel engines. Eng Fail Anal 2008;15(4):247-60.

[2] Suresh S, Sugimura Y, Tschegg EK. Growth of a Fatigue Crack Approaching a Perpendicularly Oriented Bimaterial Interface. Scripta Metall Mater 1992;27:1189-94.

[3] Pippan R, Riemelmoser FO. Fatigue of bimaterials. Investigation of the plastic mismatch in case of cracks perpendicular to the interface. Compu Mater Sci 1998;13(13):108-16.

[4] Pippan R, Flechsig K, Riemelmoser FO. Fatigue crack propagation behavior in the vicinity of an interface between materials with different yield stresses. Mater Sci Eng AStruct Mater Prop Microstruct Process 2000;283(1-2):225-33.

[5] Kolednik O, Predan J, Shan GX, Simha NK, Fischer FD. On the fracture behavior of inhomogeneous materials--A case study for elastically inhomogeneous bimaterials. Int $\mathbf{J}$ Solids Struc 2005;42(2):605-20.

[6] Wang B, Siegmund T. Simulation of fatigue crack growth at plastically mismatched bi-material interfaces. Int J Plasticity 2006;22(9):1586-609.

[7] Sugimura Y, Lim PG, Shih CF, Suresh S. Fracture normal to a bimaterial interface: Effects of plasticity on crack-tip shielding and amplification. Acta Mater 1995;43(3):115769. 
[8] Simha N, Fischer F, Kolednik O, Predan J, Shan G. Crack Tip Shielding or Antishielding due to Smooth and Discontinuous Material Inhomogeneities. Int J Fract 2005;135(1):73-93.

[9] Groh S, Olarnrithinun S, Curtin WA, Needleman A, Deshpande VS, Giessen EVd. Fatigue crack growth from a cracked elastic particle into a ductile matrix. Philos Mag 2008;88(30):3565 - 83 .

[10] Joyce MR, Reed PAS, Syngellakis S. Numerical modelling of crack shielding and deflection in a multi-layered material system. Mater Sci Eng A-Struct Mater Prop Microstruct Process 2003;342(1-2):11-22.

[11] Joyce MR, Lee KK, Syngellakis S, Reed PAS. Quantitative assessment of preferential fatigue initiation sites in a multi-phase aluminium alloy. 2004;27(11):1025-36.

[12] Chang KJ. On the maximum strain criterion--a new approach to the angled crack problem. Eng Fract Mech 1981;14(1):107-24.

[13] Sih GC. Strain Energy Density Factor Applied to Mixed Mode Crack Problems. Int J Fract 1974;10:305-21.

[14] Sih GC, Hartranft RJ. Variation of strain energy release rate with plate thickness. Int J Fract 1973; V9(1):75-82.

[15] Ali M, Syngellakis S, Reed PAS. A comparison of fatigue performance of HVOF spray coated and conventional roll bonded aluminium bearing alloys. Mat Sci Tech 2008; In Press.

[16] Burgess P. Mechanisms of sleeve bearing failure. Lubrication Engineering $1953 ; 9(309-312)$.

[17] Duckworth W, Walter G. Fatigue in plain bearings. Proceedings of the International Conference on Fatigue of Metals 1957; 1957. p. 585-92.

[18] Erdogan F, Sih GC. On the Crack Extension in Plates Under Plane Loading and Transverse Shear. J Basic Eng-Trans ASME 1963;85:519-27.

[19] Ansys. Ansys reference manual. 2005.

[20] Mwanza MC, Joyce MR, Lee KK, Syngellakis S, Reed PAS. Microstructural characterisation of fatigue crack initiation in Al-based plain bearing alloys. Int J Fatigue 2003;25(9-11):1135-45.

[21] Ali M. Microstructural modelling of fatigue in layered bearing architectures. School of Engineering Sciences. Southampton: University of Southampton; 2007. p. 275. 
[22] Barsoum R. Triangular Quarter point Elements as elastic and perfectly plastic crack tip elements. Int J Numer Methods Eng 1977;11: 85-98.

[23] McMeeking RM, Parks DM. On the Criteria for J Dominance of Crack Tip Fields in Large Scale Yielding. ASTM 1979;STP 668:175-94.

[24] Chambers AC, Hyde TH, Webster JJ. Mixed mode fatigue crack growth at 550[deg]c under plane stress conditions in Jethete M152. Eng Fract Mech 1991;39(3):603-19.

[25] Mageed AAM, Pandey RK. Mixed Mode Crack Growth under Static and Cyclic Loading in Al Alloy Sheets. Eng Fract Mech 1991;40(2):371-5.

[26] Broek D. Elementary Engineering Fracture Mechanics. 1974.

[27] Kitagawa H, Yuuki R, Ohira T. Crack-morphological aspects in fracture mechanics. Eng Fract Mech 1975;7(3):515-20.

[28] Suresh S. Micromechanisms of Fatigue Crack Growth Retardation Following Overloads. Eng Fract Mech 1983;18:577-93.

[29] Sugimura Y, Grondin L, Suresh S. Fatigue crack growth at arbitrary angles to bimaterial interfaces. Scripta Metall Mater 1995;33(12):2007-12.

[30] Burke-Veliz A, D. W, Wahdy N, Reed PAS, Merritt D, Syngellakis S. Plain bearing stresses due to forming and oil film pressure. Modern Practice in Stress and Vibration 209.

[31] Bahai $\mathrm{H}, \mathrm{Xu} \mathrm{H}$. Three-dimensional elastoplastic finite element and elastohydrodynamic analyses of journal bearings. Proc Inst Mech Eng Part C-J Eng Mech Eng Sci 1997;211:143-52. 

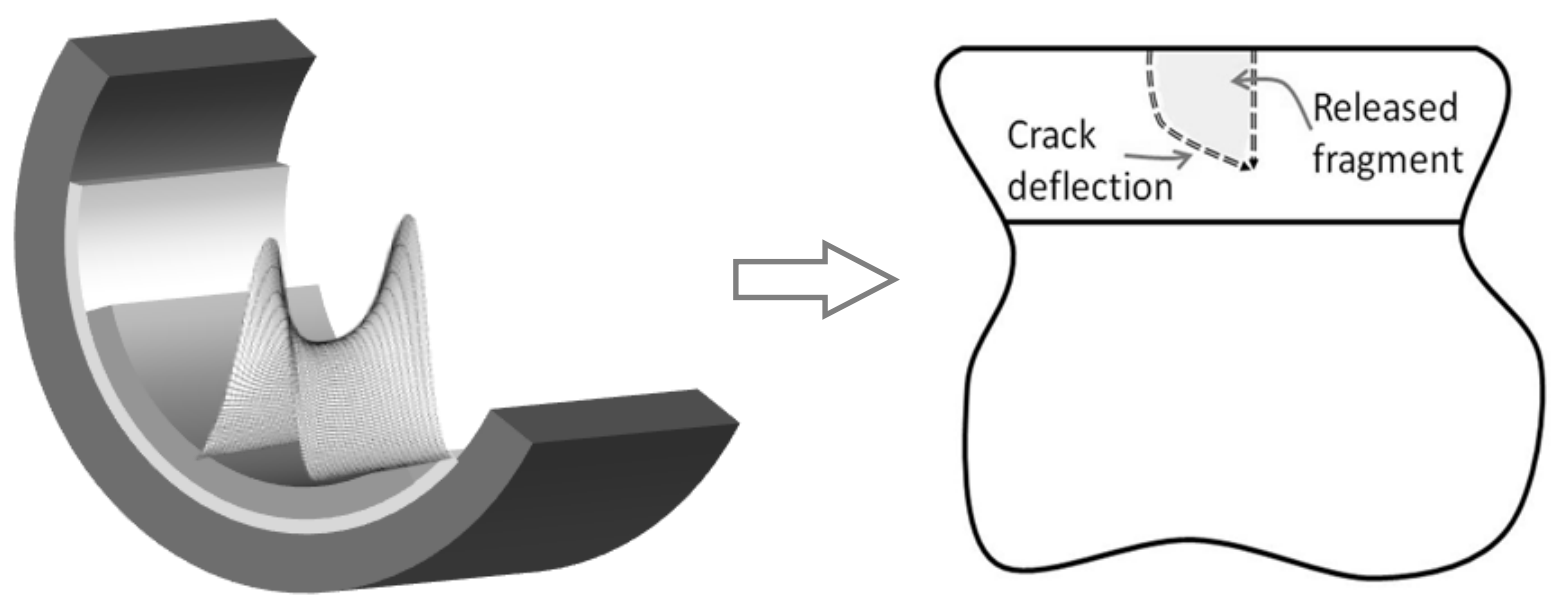

Figure 1. Multi-layered plain bearing under hydrodynamic oil film pressure and fragment release caused by fatigue fracture. 


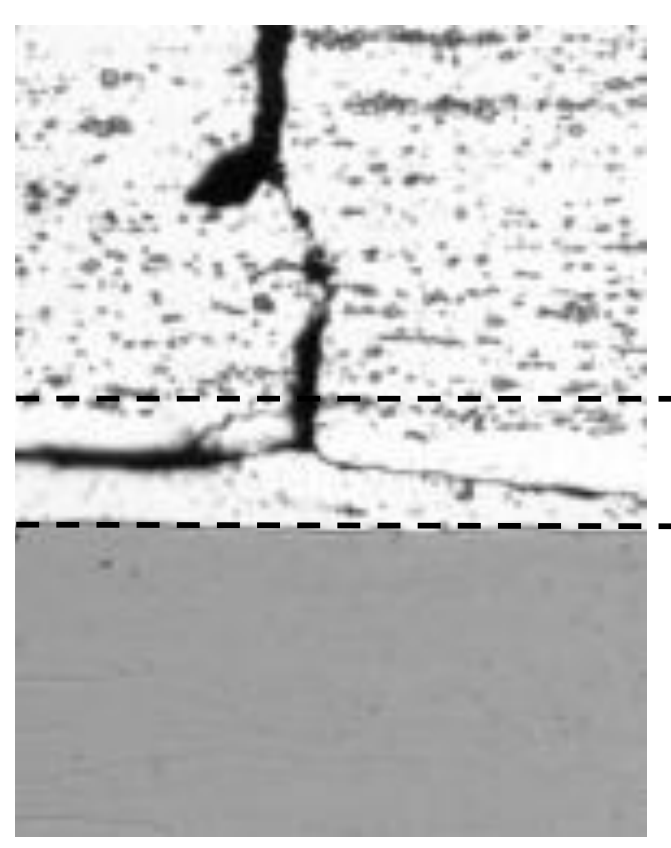

(a)

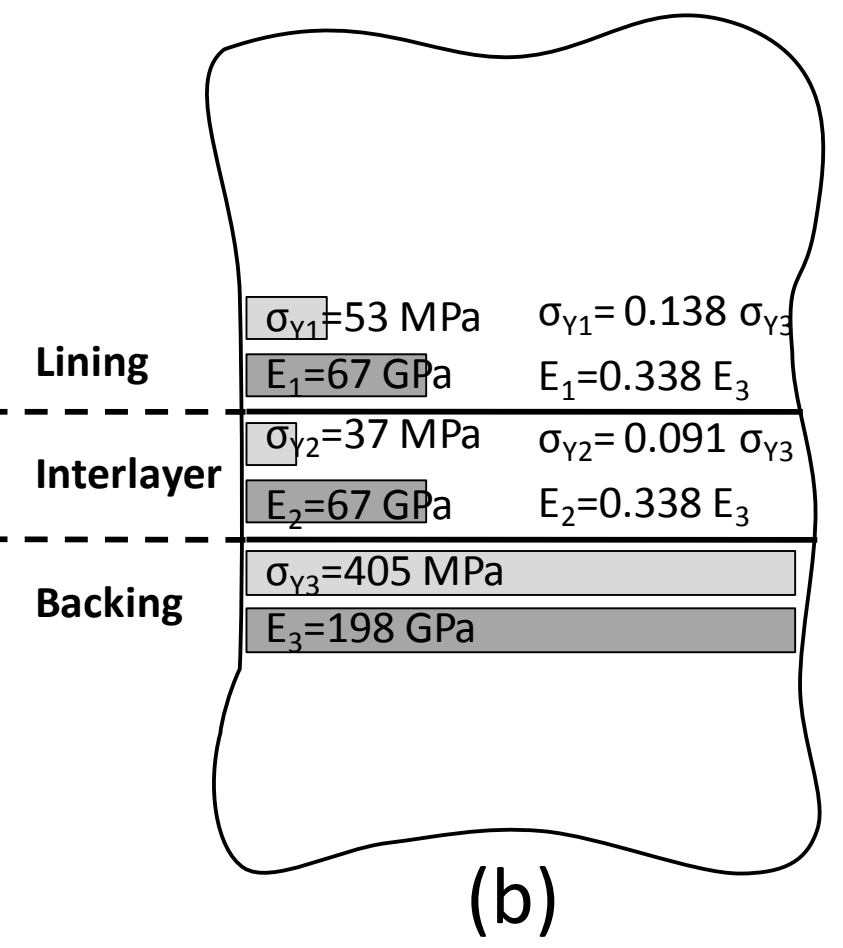

Figure 2. (a) Observed crack pattern in multi-layered architecture [3], (b) respective mechanical properties of layers. 


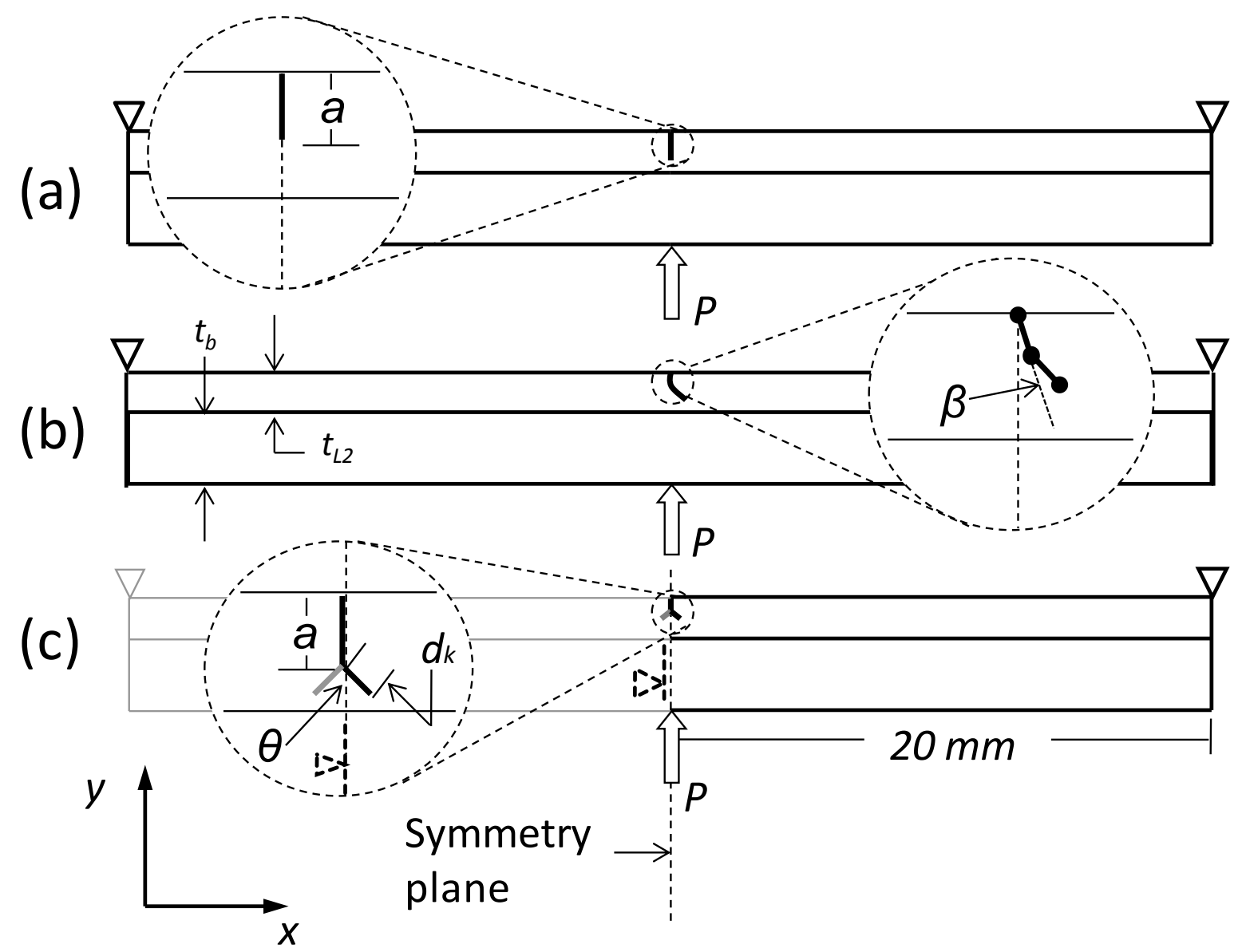

Figure 3. Schematics of analysed strip models with (a) stationary straight cracks, (b) deflected and (c) bifurcated paths. 


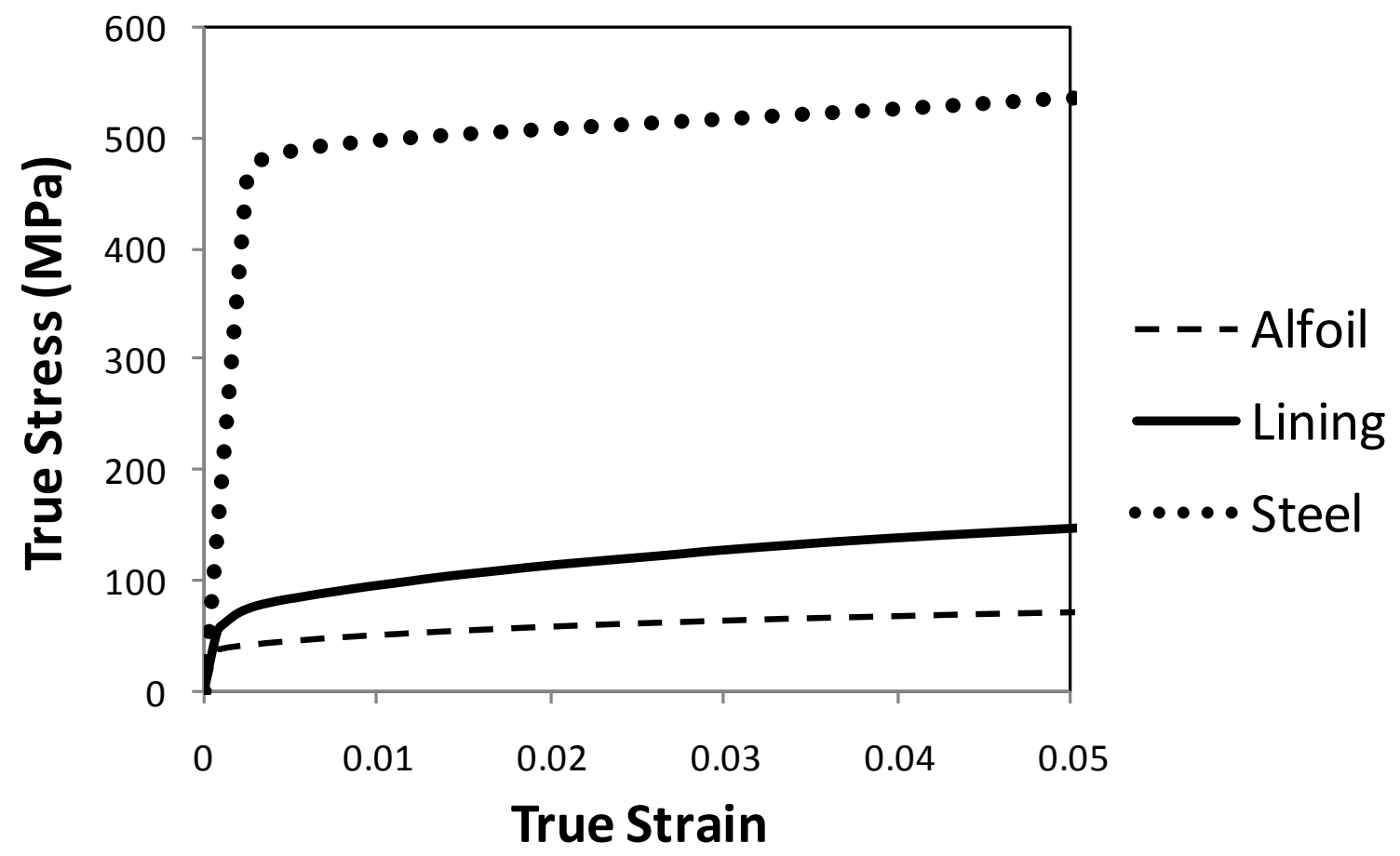

Figure 4. True stress-true strain curves for the tri-layer strip model 


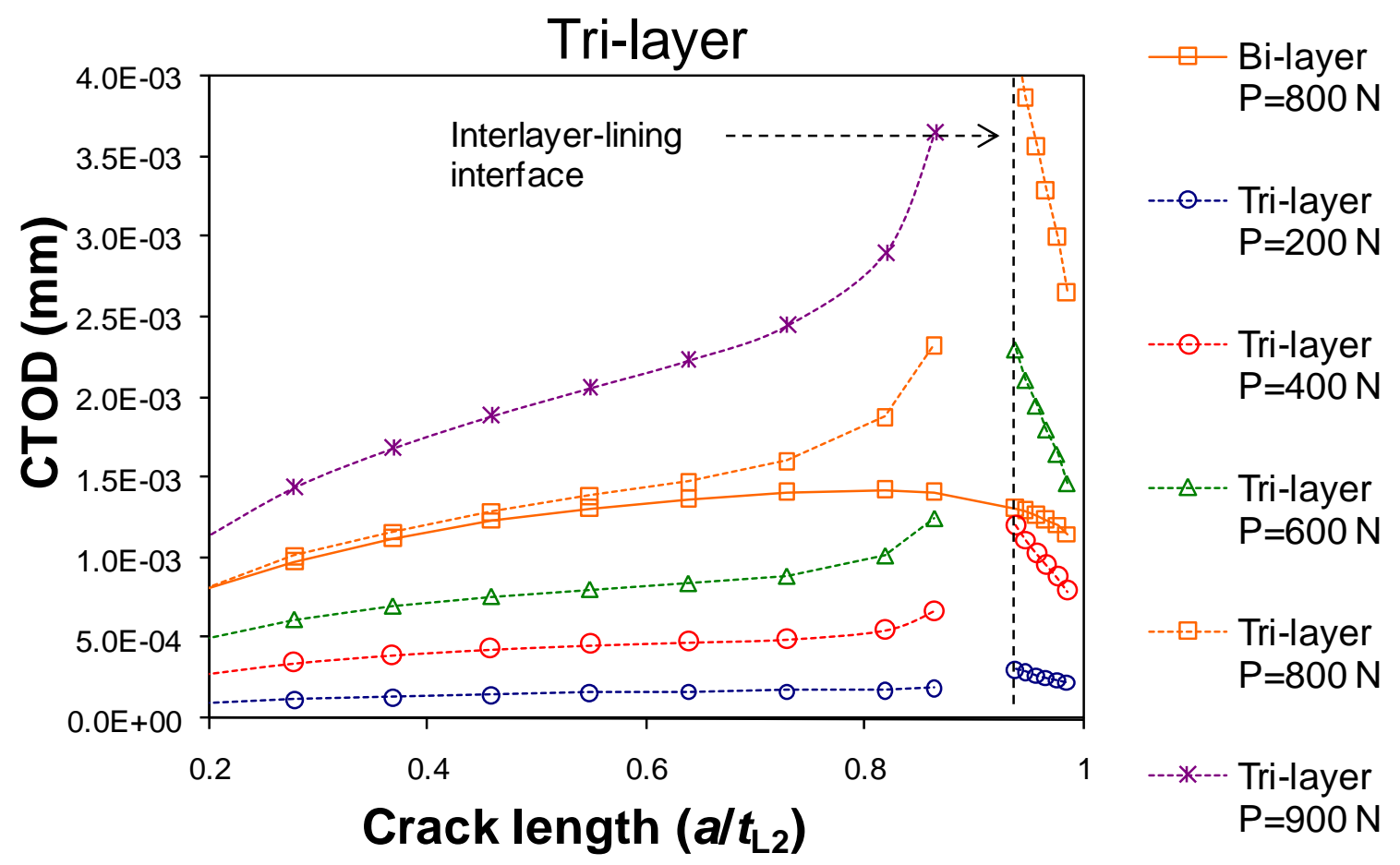

Figure 5. CTOD variation with crack length in a tri-layer strip under three-point bending 


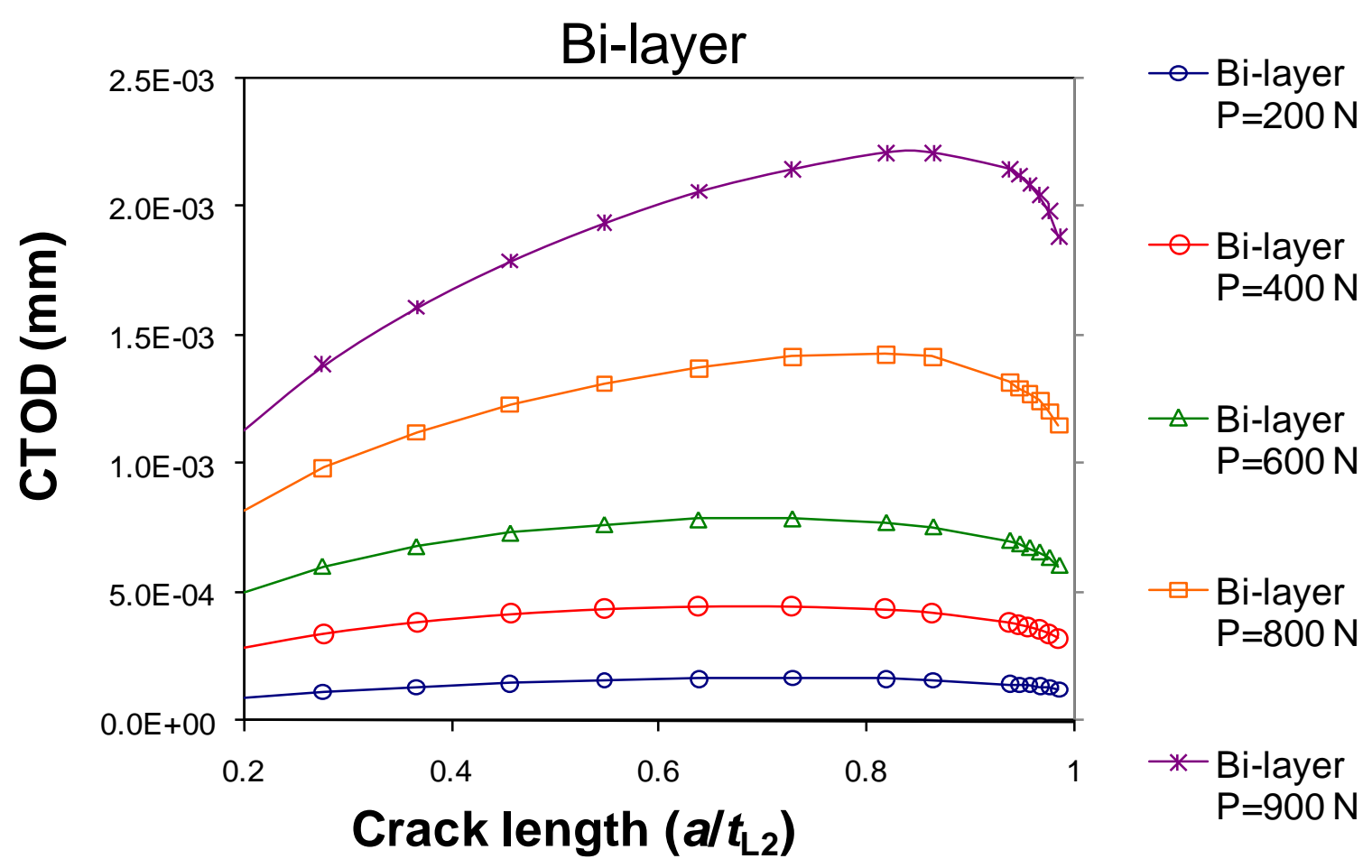

Figure 6. CTOD variation with crack length in a bi-layer strip under three-point bending 


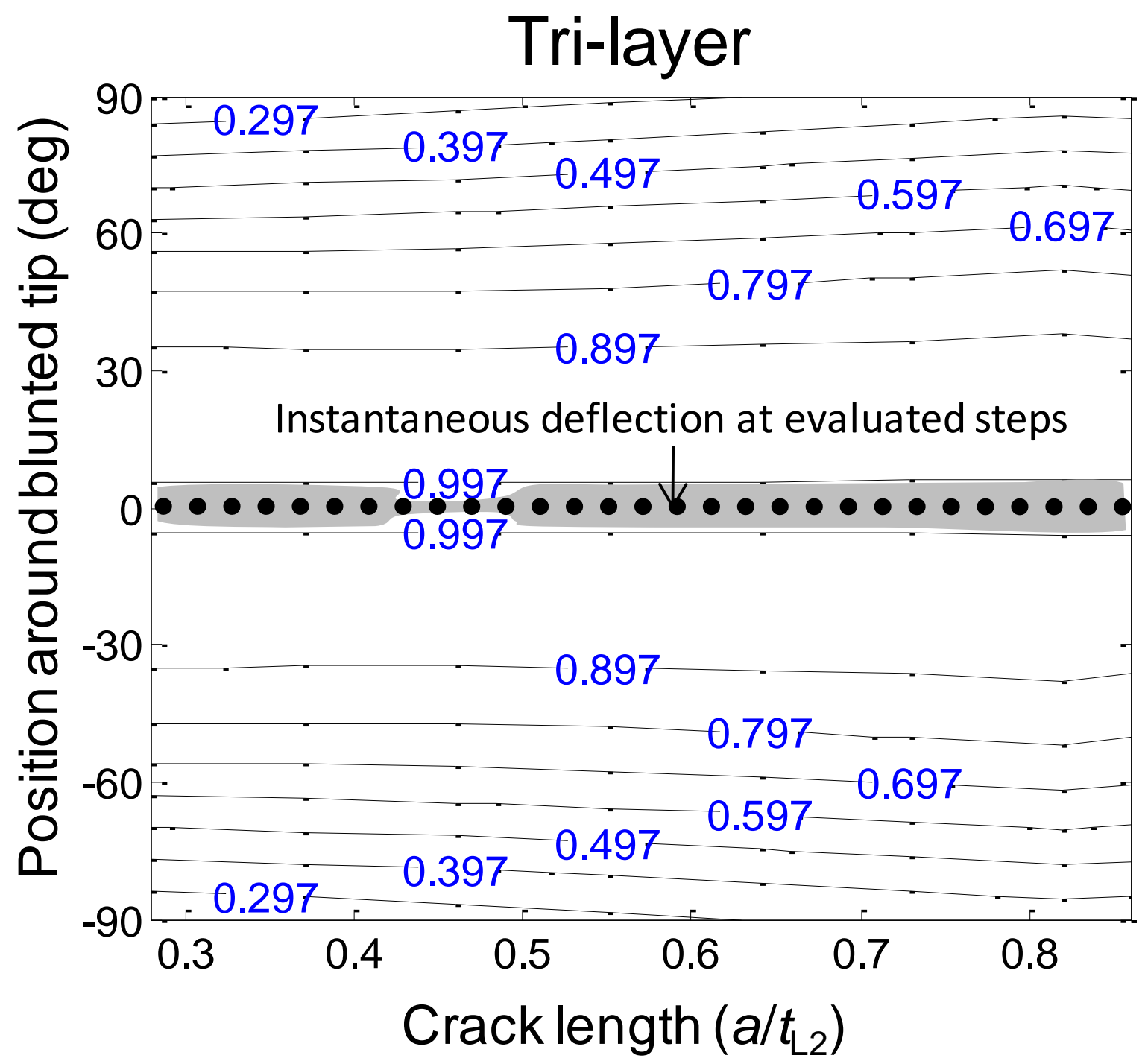

Figure 7. CTTS evolution with crack length in the tri-layer strip under $P=200 \mathrm{~N}$. 


\section{Bi-layer}

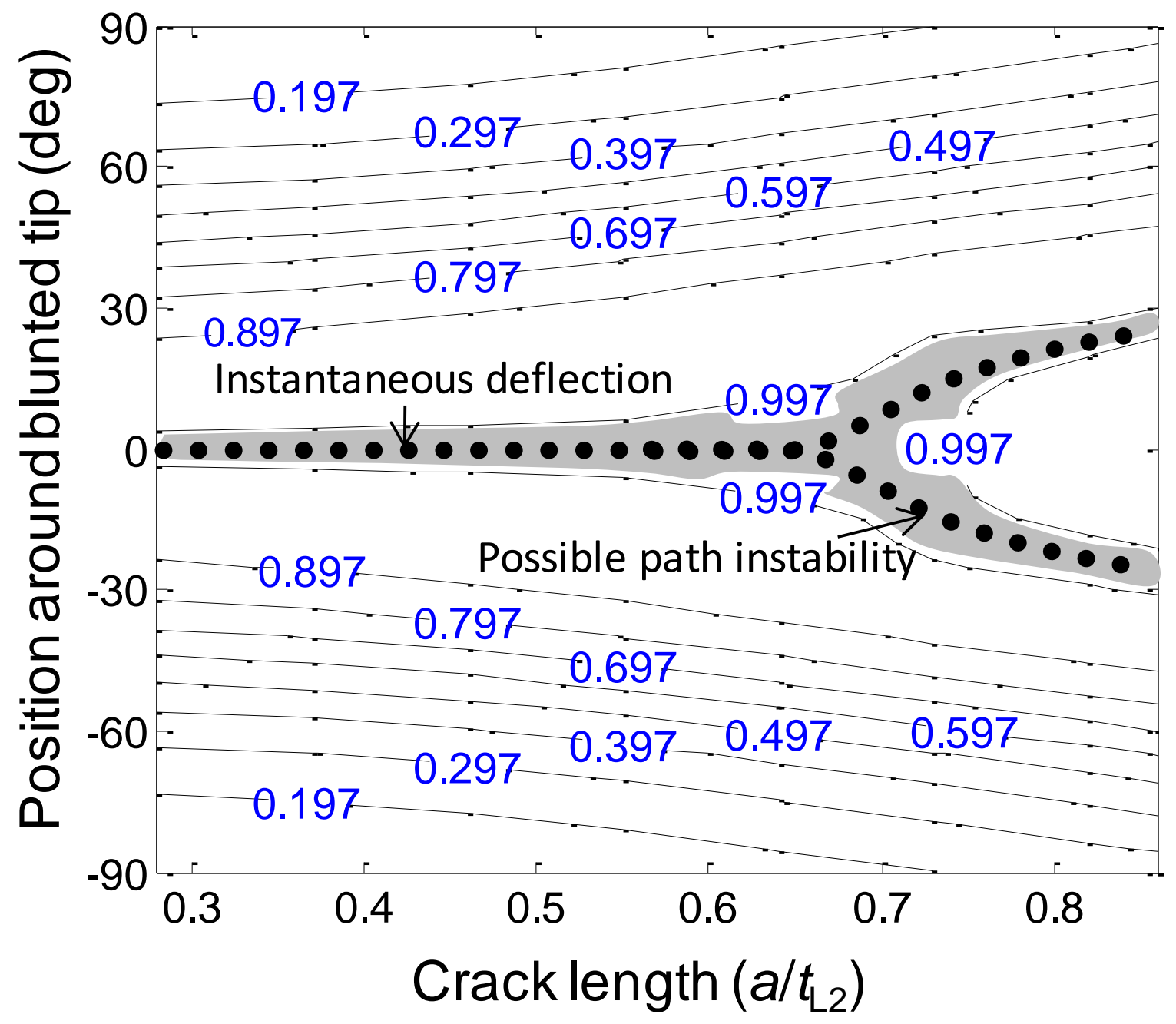

Figure 8. CTTS evolution with crack length in the bi-layer strip under $P=200 \mathrm{~N}$. 


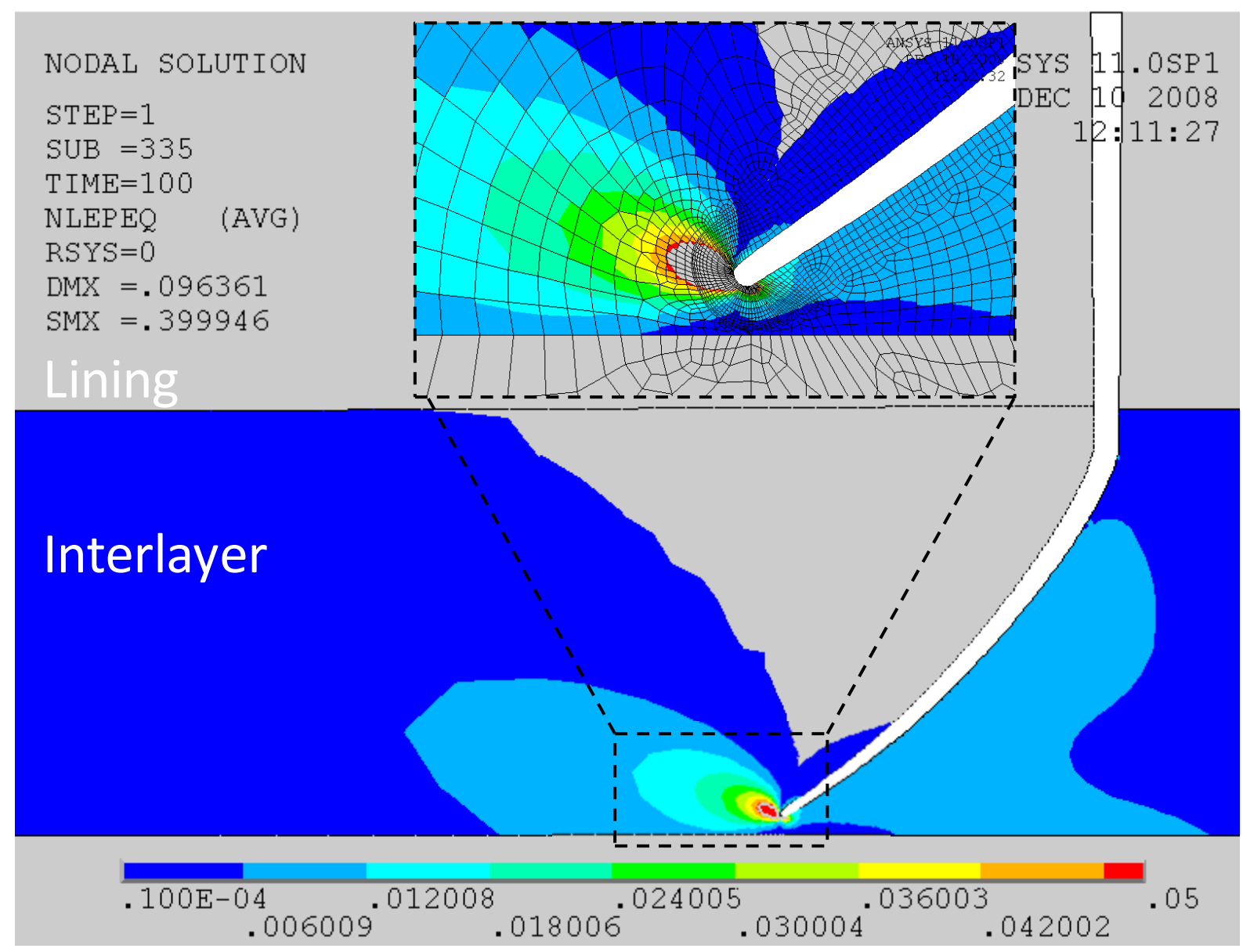

Figure 9. Deflected crack path within the lining of the tri-layer strip under $P=200 \mathrm{~N}$. 

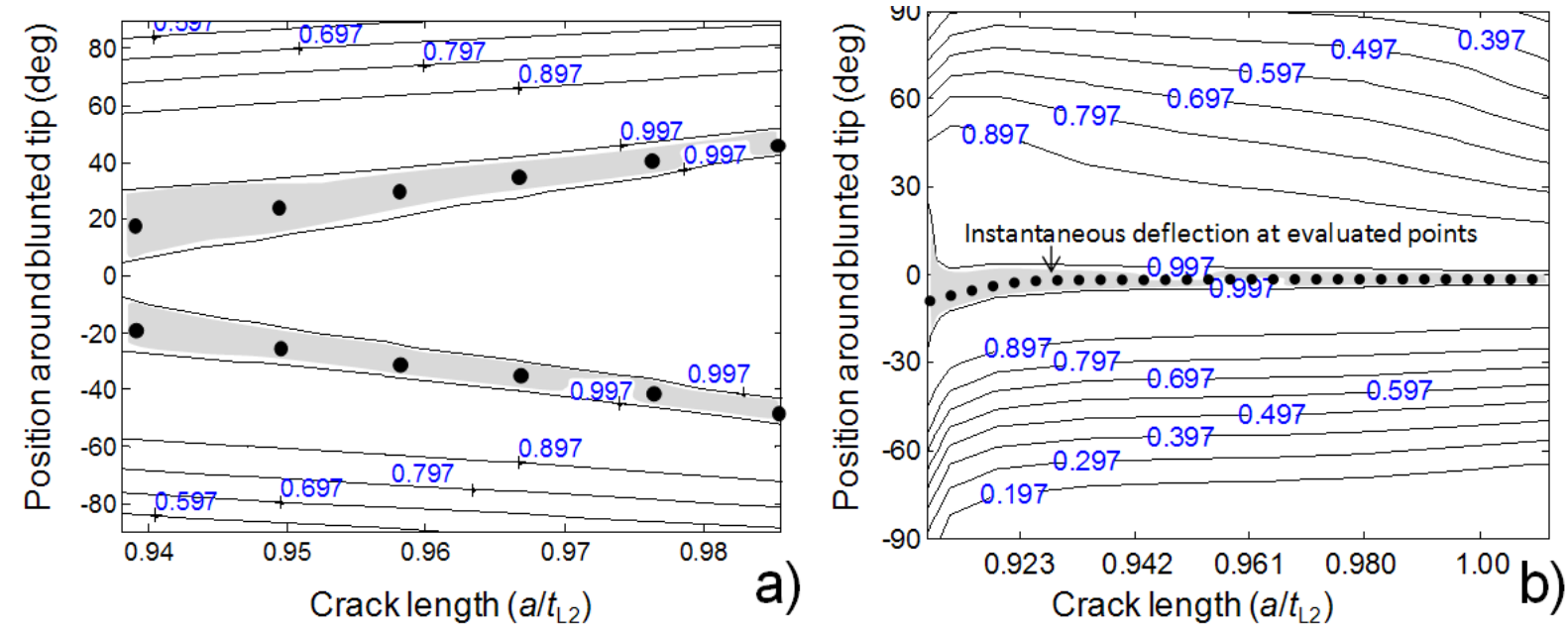

Figure 10. CTTS evolution for (a) straight and (b) deflected crack paths in the lining of the tri-layer $\operatorname{strip}(P=200 \mathrm{~N})$ 


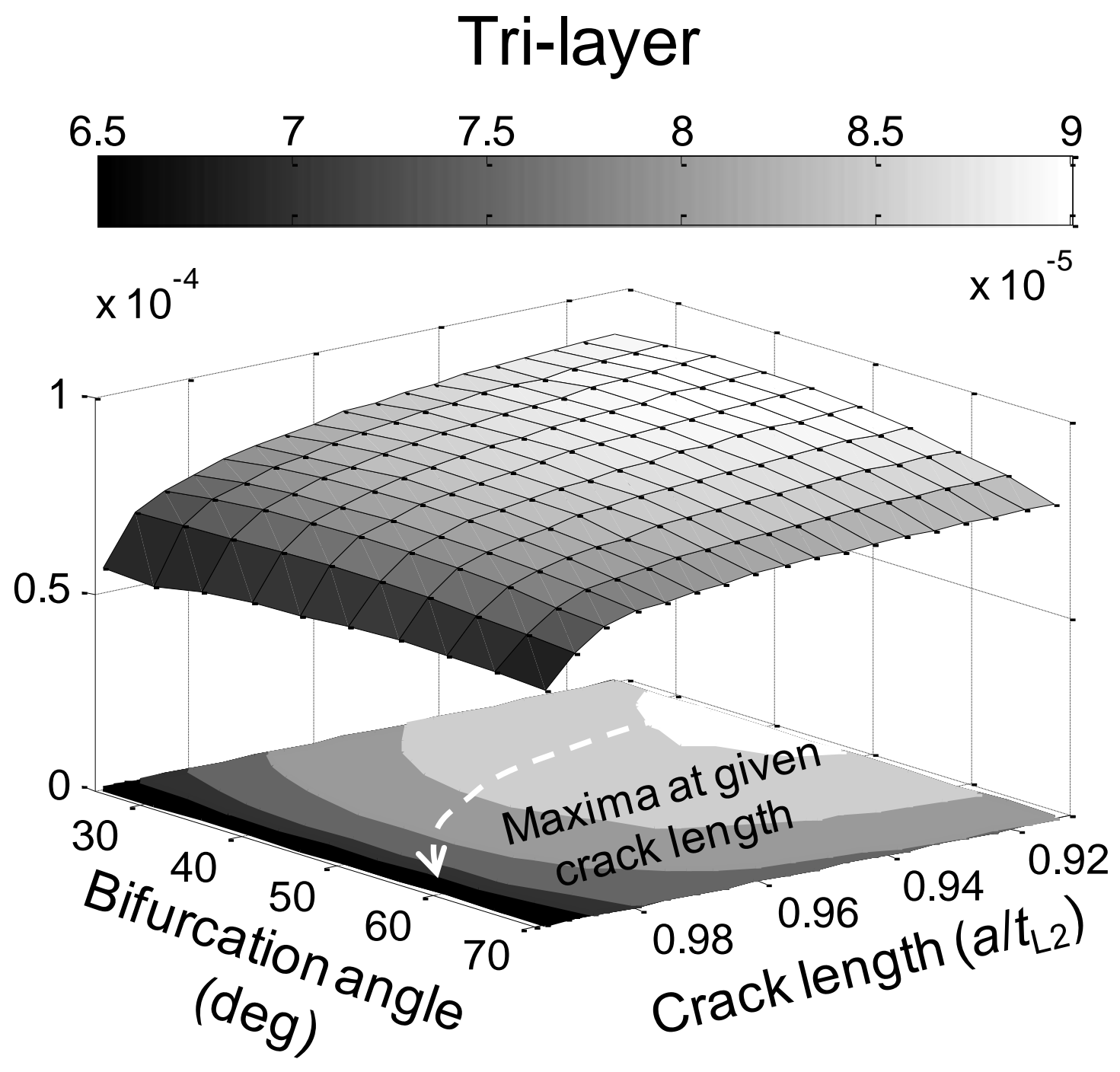

Figure 11. CTOD estimates along the bifurcated path in the tri-layer lining. 


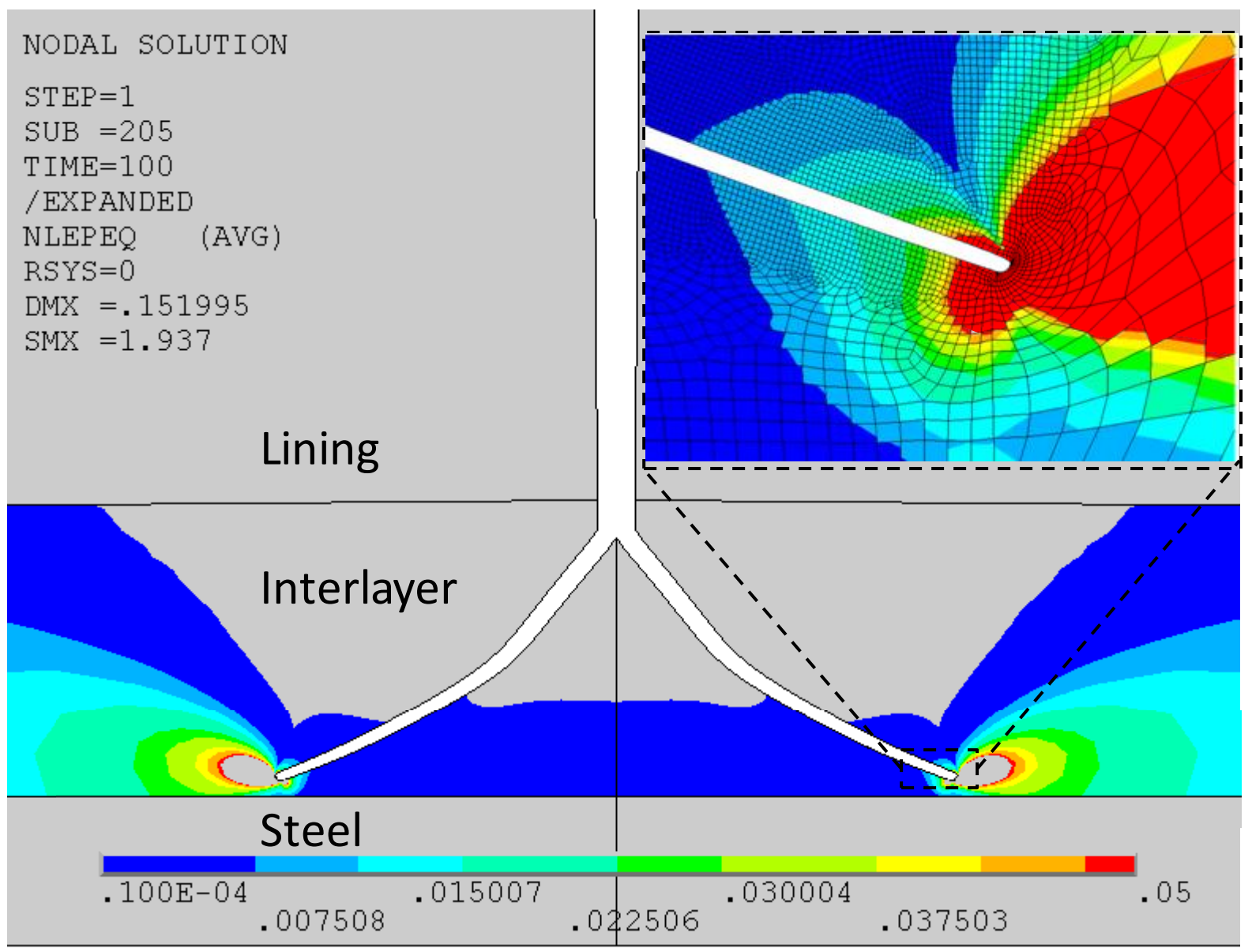

Figure 12. Bifurcated crack within the lining of the tri-layer strip with the associated equivalent plastic strain distribution 